\title{
ARITHMETIC PROPERTIES \\ OF LAGUNARY POWER SERIES WITH INTEGRAL COEFFICIENTS
}

\author{
K. MAHLER \\ (received 14 August 1964)
}

To the memory of my dear friend $J . F$. Koksma

1

This note is concerned with arithmetic properties of power series

$$
f(z)=\sum_{h=0}^{\infty} f_{h} z^{h}
$$

with integral coefficients that are lacunary in the following sense. There are two infinite sequences of integers, $\left\{r_{n}\right\}$ and $\left\{s_{n}\right\}$, satisfying

$$
0=s_{0} \leqq r_{1}<s_{1} \leqq r_{2}<s_{2} \leqq r_{3}<s_{3} \leqq \cdots, \quad \lim _{n \rightarrow \infty} \frac{s_{n}}{r_{n}}=\infty,
$$

such that

$$
f_{h}=0 \text { if } r_{n}<h<s_{n} \text {, but } f_{r_{n}} \neq 0, f_{s_{n}} \neq 0 \quad(n=1,2,3, \cdots) .
$$

It is also assumed that $f(z)$ has a positive radius of convergence, $R_{f}$ say, where naturally

$$
0<R_{f} \leqq 1 .
$$

A power series with these properties will be called admissible.

Let $f(z)$ be admissible, and let $\alpha$ be any algebraic number inside the circle of convergence,

$$
|\alpha|<R_{f}
$$

Or aim is to establish a simple test for deciding whether the value $f(\alpha)$ is an algebraic or a transcendental number. As will be found, the answer depends on the behaviour of the polynomials

$$
P_{n}(z)=\sum_{n=s_{n}}^{r_{n+1}} f_{h} z^{n} \quad(n=0,1,2, \cdots) .
$$

In terms of these polynomials, $f(z)$ allows the development

$$
f(z)=\sum_{n=0}^{\infty} P_{n}(z)
$$

which likewise converges when $|z|<R_{f}$. 


\section{2}

If

$$
a(z)=a_{0}+a_{1} z+\cdots+a_{m} z^{m}
$$

is an arbitrary polynomial, put

Then

$$
H(a)=\max _{0 \leq i \leq m}\left|a_{j}\right|, \quad L(a)=\sum_{j=0}^{m}\left|a_{j}\right| .
$$

$$
H(a b) \leqq H(a) L(b), \quad L(a b) \leqq L(a) L(b) .
$$

The following theorem is due to R. Güting (Michigan Math. J., 8 (1961), 149-159).

LEMMA 1. Let $a$ be an algebraic number which satisfies the equation

$$
A(\alpha)=0 \text {, where } A(z)=A_{0}+A_{1} z+\cdots+A_{M} z^{M} \quad\left(A_{M} \neq 0\right)
$$

is an irreducible polynomial with integral coefficients. If

$$
a(z)=a_{0}+a_{1} z+\cdots+a_{m} z^{m}
$$

is a second polynomial with integral coefficients, then either

or

$$
a(\alpha)=0
$$

$$
|a(\alpha)| \geqq\left(L(a)^{M-1} L(A)^{m}\right)^{-1}
$$

The main result of this note may be stated as follows.

THEOREM 1. Let $f(z)$ be an admissible power series, and let $\alpha$ be any algebraic number satisfying $|\alpha|<R_{f}$. The function value $f(\alpha)$ is algebraic if and only if there exists a positive integer $N=N(\alpha)$ such that

$$
P_{n}(\alpha)=0 \text { for all } n \geqq N \text {. }
$$

Corollary: If the coefficients $f_{h}$ are non-negative, then $f(z)$ is transcendental for all positive algebraic numbers $\alpha<R_{f}$. There exist, however, examples of admissible functions $f(z)$ with $f_{h} \geqq 0$ for which $S_{f}$, as defined in 4 , is everywhere dense in $|z|<R_{f}$.

Proof. It is obvious that the condition is sufficient, and so we need only show that it is also necessary.

We shall thus assume that the function value

$$
f(\alpha)=\sum_{n=0}^{\infty} f_{h} \alpha^{n}, \quad=\beta^{(0)} \text { say, }
$$

is an algebraic number, say of degree $l$ over the rational field. Let

$$
\beta^{(0)}, \beta^{(1)}, \cdots, \beta^{(l-1)}
$$


be its conjugates, and let $c_{0}$ be a positive integer such that the products

$$
c_{0} \beta^{(0)}, c_{0} \beta^{(1)}, \cdots, c_{0} \beta^{(l-1)}
$$

are algebraic integers.

We denote by $c_{1}, c_{2}, \cdots$ positive constants that may depend on $\alpha$, $\beta^{(0)}, \cdots, \beta^{(l-1)}$, but are independent of $n$. In particular, we choose $c_{1}$ such that

$$
|\alpha|<\frac{1}{c_{1}}<R_{f} \text {, hence } c_{1}>1, \quad\left|c_{1} \alpha\right|<1,
$$

and $c_{2}$ such that

$$
\left|f_{h}\right| \leqq c_{1}^{h} c_{2} \text { for all } h \geqq 0 \text {. }
$$

Put

$$
p_{n \lambda}(z)=-\beta^{(\lambda)}+\sum_{n=0}^{r_{n}} f_{h} z^{h} \quad(\lambda=0,1, \cdots, l-1)
$$

and

$$
p_{n}(z)=c_{0}^{l} \prod_{\lambda=0}^{l-1} p_{n \lambda}(z) .
$$

Then $p_{n}(z)$ is a polynomial in $z$ of degree $l r_{n}$ with integral coefficients.

From the second formula (5),

$$
L\left(p_{n}\right) \leqq c_{0}^{l} \prod_{\lambda=0}^{l-1} L\left(p_{n \lambda}\right)
$$

and here by (8) and (9),

$$
L\left(p_{n \lambda}\right) \leqq\left|\beta^{(\lambda)}\right|+\sum_{n=0}^{r_{n}}\left|f_{h}\right| \leqq c_{1}^{\tau_{n}} c_{3} \quad(\lambda=0,1, \cdots, l-1) .
$$

It follows that

$$
L\left(p_{n}\right) \leqq c_{1}^{l r_{n}} c_{4} .
$$

Since $\alpha$ is algebraic, it is the root of an irreducible equation $A(\alpha)=0$ where $A(z)$ is, say of degree $M$. On applying Lemma 1 , with $a(z)=p_{n}(z)$, we deduce from (11) that either

or

$$
p_{n}(\alpha)=0
$$

$$
\left|p_{n}(\alpha)\right| \geqq\left\{\left(c_{1}^{l r_{n}} c_{4}\right)^{M-1} L(A)^{l r_{n}}\right\}^{-1} \geqq c_{5}^{-l r_{n}} .
$$

However, the second alternative (12) cannot hold if $n$ is sufficiently large. For by (6), (9), and (10), 


$$
\left|p_{n 0}(\alpha)\right|=\left|\sum_{h=s_{n}}^{\infty} f_{h} \alpha^{h}\right| \leqq\left|c_{1} \alpha\right|^{s_{n}} c_{6} \text {, }
$$

and it is also obvious that

$$
\left|p_{n \lambda}(\alpha)\right| \leqq c_{7} \quad(\lambda=1,2, \cdots, l-1) .
$$

On combining these estimates it follows that

$$
\left|p_{n}(\alpha)\right| \leqq c_{0}^{l} \cdot\left|c_{1} \alpha\right|^{s_{n}} c_{6} \cdot c_{7}^{l-1}<c_{5}^{-l r_{n}}
$$

for all sufficiently large $n$, because by (1) and (8),

$$
\left|c_{1} \alpha\right|<1, \quad \lim _{n \rightarrow \infty} \frac{s_{n}}{r_{n}}=\infty .
$$

Thus there exists an integer $N_{0}$ such that

$$
p_{n}(\alpha)=0 \text { for all } n \geqq N_{0} \text {. }
$$

This means that to every integer $n \geqq N_{0}$ there exists a suffix $\lambda_{n}$ which has one of the values $0,1,2, \cdots, l-1$ such that

$$
\sum_{h=0}^{r_{n}} f_{h} \alpha^{h}=\beta^{\left(\lambda_{n}\right)}
$$

Therefore also

$$
P_{n}(\alpha)=\sum_{h=0}^{r_{n+1}} f_{h} \alpha^{h}-\sum_{h=0}^{r_{n}} f_{h} \alpha^{h}=\beta^{\left(\lambda_{n+1}\right)}-\beta^{\left(\lambda_{n}\right)} \quad \text { if } \quad n \geqq N_{0} .
$$

Now $f(\alpha)$ is a convergent series, and hence

$$
\lim _{n \rightarrow \infty} P_{n}(\alpha)=0
$$

On the other hand, the $l$ conjugate numbers (7) are all distinct. There is then an integer $N \geqq N_{0}$ with the property that

$$
\lambda_{n+1}=\lambda_{n} \quad \text { if } n \geqq N .
$$

By (13), this implies that

$$
P_{n}(\alpha)=0 \text { if } n \geqq N,
$$

giving the assertion.

\section{4}

Let $\Sigma$ be a set of algebraic numbers, $S$ a subset of $\Sigma$. For each element $\alpha$ of $\Sigma$ denote by $A(\alpha)$ the set of all algebraic conjugates $\alpha, \alpha^{\prime}, \alpha^{\prime \prime}, \cdots$ of $\alpha$ that belong to $\Sigma$. We say that the set $S$ is complete relative to $\Sigma$ if 
$\alpha \in S$ implies that also $A(\alpha) \in S$.

Let again $f(z)$ be an admissible power series. Then denote by $\Sigma_{f}$ the set of all algebraic numbers $\alpha$ satisfying $|\alpha|<R_{f}$ and by $S_{f}$ the set of all $\alpha \in \Sigma_{f}$ for which $f(\alpha)$ is algebraic.

THEOREM 2. If $f(z)$ is admissible, the set $S_{f}$ is complete relative to $\Sigma_{f}$.

Proof. Let $\alpha$ be any element of $S_{f}$, and let $q(z)$ be the primitive irreducible polynomial with integral coefficients and positive highest coefficient for which $q(\alpha)=0$. By Theorem 1,

and hence

$$
P_{n}(\alpha)=0 \text { for } n \geqq N \text {, }
$$

$P_{n}(z)$ is divisible by $q(z)$ for all suffixes $n \geqq N$.

Hence, if $\alpha^{\prime}$ is any conjugate of $\alpha$, also

$$
P_{n}\left(\alpha^{\prime}\right)=0 \text { for } n \geqq N .
$$

Assume, in particular, that $\alpha^{\prime} \in \Sigma_{f}$, hence that $f\left(\alpha^{\prime}\right)$ converges. Then, by Theorem $1, f\left(\alpha^{\prime}\right)$ is algebraic, and therefore also $\alpha^{\prime}$ is in $S_{f}$.

The following result establishes all possible sets $S_{f}$ in which an admissible power series can assume algebraic values.

THEOREM 3. Let $R$ be a positive constant not greater than 1 ; let $\Sigma$ be the set of all algebraic numbers $\alpha$ satisfying $|\alpha|<R$; and let $S$ be any subset of $\Sigma$ which contains the element 0 and is complete relative to $\Sigma$. Then there exists an admissible power series $f(z)$ with the property that

$$
R_{f}=R \text { and } S_{f}=S .
$$

Proof. As a set of algebraic numbers, $S$ is countable. It is therefore possible to define an infinite sequence of polynomials

$$
\left\{q_{n}(z)\right\}=\left\{q_{0}(z), q_{1}(z), q_{2}(z), \cdots\right\}
$$

with the following properties.

If $S$ consists of the single element 0 , put $q_{n}(z) \equiv \mathbf{1}$ for all suffixes $n$. If $S$ is a finite set, take for the first finitely many elements of $\left\{q_{n}(z)\right\}$ all distinct primitive irreducible polynomials with integral coefficients and positive highest coefficients that vanish in at least one point $\alpha$ of $S$, and put all remaining sequence elements equal to $q_{n}(z) \equiv 1$. If, finally, $S$ is an infinite set, let $\left\{q_{n}(z)\right\}$ consist of all distinct primitive irreducible poly- 
nomials with integral coefficients and positive highest coefficients that vanish in at least one point $\alpha$ of $S$.

Further let

$$
Q_{n}(z)=q_{0}(z) q_{1}(z) \cdots q_{n}(z) \quad(n=0,1,2, \cdots) ;
$$

denote by $d_{n}$ the degree of $Q_{n}(z)$; and put

$$
H_{n}=H\left(Q_{n}\right) \quad(n=0,1,2, \cdots) .
$$

Next choose a sequence of integers $\left\{s_{n}\right\}$ where

such that

$$
0=s_{0}<s_{1}<s_{2}<\cdots
$$

$$
\lim _{n \rightarrow \infty} \frac{s_{n}}{d_{n}}=\infty, \quad \lim _{n \rightarrow \infty} \frac{s_{n+1}}{s_{n}}=\infty, \quad \lim _{n \rightarrow \infty} H_{n}^{1 / s_{n}}=1
$$

and

Hence, on putting

$$
s_{n+1}>s_{n}+d_{n} \quad(n=0,1,2, \cdots) .
$$

$$
r_{n+1}=s_{n}+d_{n} \quad(n=0,1,2, \cdots),
$$

the two sequences $\left\{r_{n}\right\}$ and $\left\{s_{n}\right\}$ have the property

$$
0=s_{0} \leqq r_{1}<s_{1} \leqq r_{2}<s_{2} \leqq r_{3}<s_{3} \leqq \cdots, \lim _{n \rightarrow \infty} \frac{s_{n}}{r_{n}}=\infty
$$

Finally denote by $\left\{K_{n}\right\}$ a sequence of positive integers satisfying

$$
\lim _{n \rightarrow \infty} K_{n}^{1 / s_{n}}=\frac{1}{R}
$$

On putting

$$
P_{n}(z)=K_{n} Q_{n}(z) z^{s_{n}},=\sum_{n=s_{n}}^{r_{n+1}} f_{h} z^{h} \text { say } \quad(n=0,1,2, \cdots),
$$

and

$$
f(z)=\sum_{n=0}^{\infty} P_{n}(z)=\sum_{h=0}^{\infty} f_{h} z^{n},
$$

$f(z)$ is a lacunary power series of the kind defined in $\S 1$.

Distinct polynomials $P_{n}(z)$ evidently involve different powers of $z$, so that the contributions to $f(z)$ from these polynomials do not overlap.

To prove that $f(z)$ is admissible we have to prove that the radius $R_{f}$ of convergence of $f(z)$ is positive. In fact

$$
\frac{1}{R_{f}}=\limsup _{h \rightarrow \infty}\left|f_{h}\right|^{1 / n}
$$

and this, by the formulae (1) and (14), is equal to 
Further

$$
\frac{1}{R_{f}}=\lim _{\substack{s_{n} \leqq h \leqq r_{n+1} \\ n \rightarrow \infty}}\left|f_{h}\right|^{1 / s_{n}} .
$$

$$
\left|f_{h}\right| \leqq H_{n} K_{n} \text { for } s_{n} \leqq h \leqq r_{n+1},
$$

with equality for at least one suffix $h$ in this interval. Hence, by (14) and (15),

so that

$$
\frac{1}{R_{f}}=\limsup _{n \rightarrow \infty}\left(H_{n} K_{n}\right)^{1 / s_{n}}=\frac{1}{R}
$$

The second assertion

$$
R_{f}=R>0
$$

$$
S_{f}=S
$$

is now an immediate consequence of Theorem 1 and the construction of the polynomials $P_{n}(z)$. For if $\alpha$ is any element of $S$, then evidently $P_{n}(z)$, for sufficiently large $n$, will be divisible by the polynomial $q_{\nu}(z)$ which has $\alpha$ as a root, and so $\alpha \in S_{f}$. On the other hand, if $\alpha$ is not an element of $S$, no polynomial $q_{\nu}(z)$ and hence also no polynomial $P_{n}(z)$ vanishes for $z=\alpha$.

\section{6}

The two Theorems 1 and 3 together solve the problem of establishing all possible sets $S_{f}$ in which an admissible function may be algebraic. In order to obtain further results, it becomes necessary to specialise $f(z)$.

Let us, in particular, consider those admissible power series

$$
f(z)=\sum_{n=0}^{\infty} f_{h} z^{n}
$$

which are of the bounded type, i.e. to which there exists a positive constant $c$ such that

$$
\left|f_{h}\right| \leqq c \text { for all } h \geqq 0 .
$$

For such series the set $S_{f}$ is restricted as follows.

THEOREM 4. If $f(z)$ is an admissible power series of the bounded type, then $S_{f}$ may, or may not, be an infinite set. If

is an infinite set, then

$$
S_{f}=\left\{\alpha_{1}, \alpha_{2}, \alpha_{3}, \cdots\right\}
$$

$$
\lim _{k \rightarrow \infty}\left|\alpha_{k}\right|=R_{f}=1 \text {. }
$$

Proof. (i) It is obvious from Theorem 1 that there exist admissible power series of the bounded type for which $S_{f}$ is a finite set, e.g. consists 
of the single point 0 . The following construction, on the other hand, leads to such a series for which $S_{f}$ is an infinite set.

We procede similarly as in the proof of Theorem 3, but take $R=1$ and

$$
q_{n}(z)=1-z^{3^{n}}-z^{2 \cdot 3^{n}}, \quad K_{n}=1 \quad(n=0,1,2, \cdots) .
$$

Then, in the former notation,

$$
H_{n}=1 \quad(n=0,1,2, \cdots),
$$

because the Taylor coefficients of $Q_{n}(z)=q_{0}(z) q_{1}(z) \cdots q_{n}(z)$ all can only be equal to $0,+1$, or -1 . The construction leads therefore to an admissible power series $f(z)$ the Taylor coefficients of which likewise can only be equal to $0,+1$, or -1 . Furthermore, the corresponding set $S_{f}$ consists of the infinitely many numbers

$$
\sqrt[3^{n}]{\frac{\sqrt{5-1}}{2}}
$$$$
(n=0,1,2, \cdots) \text {. }
$$

(ii). Next let $f(z)$ be an admissible power series of the bounded type, thus with the radius of convergence $R_{f}=1$, and let $r$ and $R$ be any two constants satisfying

$$
0<r<R<1 .
$$

Let $S_{f}(r)$ be the subset of those elements $\alpha$ of $S_{f}$ for which

$$
|\alpha| \leqq r .
$$

We apply again the formulae (3) and (4) and put

here, by (2),

$$
P_{n}^{*}(z)=z^{-s_{n}} P_{n}(z)=\sum_{n=s_{n}}^{r_{n+1}} f_{h} z^{h-s_{n}} \quad(n=1,2,3, \cdots)
$$

$$
P_{n}^{*}(0)=f_{s_{n}} \neq 0 \quad(n=1,2,3, \cdots) .
$$

Therefore, by Jensen's formula,

$$
\sum_{\alpha} \log \frac{R}{|\alpha|}=\log \frac{1}{\left|f_{s_{n}}\right|}+\frac{1}{2 \pi} \int_{0}^{2 \pi} \log \left|P_{n}^{*}\left(R e^{\vartheta i}\right)\right| d \vartheta,
$$

where $\sum_{\alpha}$ extends over all zeros $\alpha$ of $P_{n}^{*}(z)$ for which $|\alpha| \leqq R$. Here, on the right-hand side,

$$
\log \frac{1}{\left|f_{s_{n}}\right|} \leqq 0, \quad\left|P_{n}^{*}\left(R e^{\vartheta i}\right)\right| \leqq c\left(1+R+R^{2}+\cdots\right)=\frac{c}{1-R} \text { for real } \vartheta,
$$

where $c$ is the constant in (16). 
Assnme, in particular, that $|\alpha| \leqq r$ and hence $\log R /|\alpha| \geqq \log R / r$. The inequality (17) shows then that $P_{n}^{*}(z)$ cannot have more than

$$
\left(\log \frac{c}{1-R}\right) /\left(\log \frac{R}{r}\right)
$$

zeros for which $|\alpha| \leqq r$. This estimate is independent on $n$. On allowing both $R$ and $r$ to tend to 1 , the assertion follows immediately from Theorem 1.

Mathematics Department, Institute of Advanced Studies, Australian National University, Canberra, A.C.T.

11th August, 1964 